\title{
Entrelacs
}

Cinéma et audiovisuel

Nouvelles formes audiovisuelles documentaires

\section{Audiostories, retour sur la création collective d'une forme audiovisuelle « ouverte " pour le web.}

Amanda Robles and Audrey Ginestet et Fabrice Lapeyrere.

\section{OpenEdition}

\section{Journals}

\section{Electronic version}

URL: http://journals.openedition.org/entrelacs/1882

DOI: 10.4000/entrelacs.1882

ISSN: 2261-5482

\section{Publisher}

Éditions Téraèdre

\section{Electronic reference}

Amanda Robles and Audrey Ginestet et Fabrice Lapeyrere., " Audiostories, retour sur la création collective d'une forme audiovisuelle « ouverte » pour le web. », Entrelacs [Online], 12 | 2016, Online since 14 January 2016, connection on 01 May 2019. URL : http://journals.openedition.org/ entrelacs/1882 ; DOI : 10.4000/entrelacs.1882

This text was automatically generated on 1 May 2019.

Tous droits réservés 


\section{Audiostories, retour sur la création collective d'une forme audiovisuelle « ouverte » pour le web.}

Amanda Robles and Audrey Ginestet et Fabrice Lapeyrere.

1 Imaginer quelque chose qui n'existe pas. Imaginer un objet audiovisuel qui ne soit pas tout à fait un film ni vraiment un web-documentaire. Un objet fait d'images et de sons comme un point de rencontre entre un photomontage et une émission radio. Une forme audiovisuelle ouverte qui revendique son hybridité et sa fragmentation. Des images d'un côté, des sons de l'autre. Entre eux des possibilités de contact et de connexion mais aussi la coprésence de mondes autonomes dont on ne viendrait pas forcer les points de rencontre ou les rapports de force, les correspondances ou les écarts.

2 Les enjeux du projet web audiostories.fr, dont l'élaboration a duré plus de deux ans, se sont dès l'origine concentrés sur cette possibilité de disjoindre le son des images. En effet, ce qui nous apparaissait comme l'une des spécificités les plus novatrices du web était la fragmentation quasi illimitée des formes et des contenus. Monde en morceaux, constellations de données et de subjectivités, le web s'affiche comme un espace ouvert aux tentatives et tâtonnements, objets inachevés et formes évolutives. Or la délinéarisation proposée par les webdocs propose le plus souvent de fragmenter des contenus selon des «chapitres » ou des « zones » répondant à une organisation logique (donc linéaire à l'origine ${ }^{1}$ ) mais rarement de faire éclater la forme même de ces contenus. L'idée est souvent d'agencer de manière originale des vidéos, des images fixes, des sons et des textes mais rarement d'inventer d'autres façons de les écouter, de les regarder, de les lire ou de les faire interagir entre eux.

Désirant réaliser une série de portraits documentaires sur les savoir-faire auditifs, nous nous sommes longtemps interrogés sur la forme audiovisuelle qui permettrait de donner le mieux à entendre ces témoignages intimes, ténus, parfois difficilement exprimables. Un berger qui choisit les sonnailles de son troupeau pour faire un accord majeur, une infirmière qui travaille au rythme des «bip-bip» de l'hôpital, une vieille dame qui se souvient des chansons qu'elle écoutait quand elle était jeune, un animateur nature qui 
organise des sorties nocturnes pour écouter le brame du cerf, un artiste plasticien qui construit des installations sonores, un cuisinier qui tend l'oreille pour préparer ses plats... Ces expériences auditives subjectives nous semblaient devoir être recueillies avec simplicité, préservées dans leur fragilité, et partagées de manière originale.

Nous allons ici essayer de retracer pourquoi et comment nous avons été amenés à concevoir un nouveau format audiovisuel pour le web et pour quelles raisons nous avons souhaité en faire une forme " ouverte ». Ouverte parce qu'elle propose une mise à plat des contenus et une tentative de rupture avec le caractère fondamentalement agglomérant du montage mais aussi parce qu'elle a été pensée comme un outil à partager. En effet, l'aspect participatif du projet a évolué au fil du temps allant jusqu'à la création d'une interface de montage et de publication en ligne utilisable par tous.

\section{Fragmenter le documentaire}

5 A l'origine, l'idée était d'utiliser le web comme simple média de diffusion pour publier régulièrement de courts portraits documentaires. La brièveté des contenus audiovisuels rencontrés sur internet et la fantaisie des agencements entre ces matériaux nous semblaient ouvrir de nouvelles portes à la création documentaire. Combien de fois avions-nous été touchés par une personne sans nous décider pour autant à nous engager dans un projet de film? Nous commencions à imaginer un espace virtuel qui nous permette de publier rapidement, au fur et à mesure de nos rencontres, du contenu documentaire et de mettre en commun ces travaux grâce à une charte précise de réalisation et de mise en forme.

6 Le projet était né au départ d'un élan collectif et nous souhaitions ouvrir le site à la participation d'autres réalisateurs afin que la série puise s'enrichir de nouveaux points de vue. La série des 24 Portraits qu'Alain Cavalier avait réalisée pour la télévision entre 1987 et 1992 avait toujours représentée une forte source d'inspiration et la découverte du projet itinérant produit par David Lynch, The Interview project nous avait fait mesurer combien de nouvelles formes audiovisuelles restaient à imaginer pour construire des passerelles entre le portrait documentaire et les potentialités du web ${ }^{2}$. Plusieurs POM (Petites Oeuvres Multimédia) ont attiré notre attention par leur tentative de renouveler les relations image/son au sein d'objets audiovisuels documentaires très simples, souvent proches du diaporama. Nous citerons en particulier le projet Histoires courtes (http:// histoires-courtes.fr) réalisé par Jean-François Dars et Anne Papillault, une série de portraits de chercheurs en noir et blanc accompagnés d'entretiens sonores de trois minutes ou encore One in 8 million (http://www.nytimes.com/packages/html/nyregion/1in-8-million), galerie de portraits de new-yorkais pour le site internet du New York Times.

7 Dans ces deux propositions, la grande brièveté des films (entre trois et quatre minutes) est accentuée par l'aspect fragmentaire de la photographie. Le portrait documentaire se fait impressionniste : la durée se construit d'instant en instant et le portrait s'esquisse détail après détail. Une série de moments, une suite de morceaux de corps, de lieux et d'objets. Par cette fragmentation l'image se fait moins totalisante et les gouffres laissés dans les intervalles entre chaque cliché permettent de mieux laisser entendre la présence des voix et des sons. Ainsi ce monde disjoint et elliptique est en quelque sorte reconstruit et solidifié par le fil de la voix qui nous raconte l'histoire secrète des fragments. Un autre commentaire, une autre voix et l'on pourrait y voir tout à fait autre chose ! Il pourrait même s'agir de la voix de quelqu'un d'autre, au fond qu'en saurions-nous? Le point de 
synchronisme et de congruence n'est pas donné. Les deux mondes sont dissociés mais s'attirent irrésistiblement tandis que ce jeu sur les différents intervalles (entre chaque cliché mais aussi entre les sons et les images) donne à l'ensemble une fragilité qui nous semblait particulièrement émouvante.

Nous ressentions pourtant une gêne face au rapport inévitablement illustratif entre l'entretien sonore et les photographies. Nous avons alors décidé de déconstruire ce dispositif par deux moyens : d'une part en interrompant le flux des images et d'autre part en fragmentant l'espace réservé aux photographies. Nous avons ainsi imaginé laisser à l'internaute le soin de faire défiler ou non les images et que celles-ci apparaissent non pas une à une mais sous forme de paires. La paire permettait en effet de défaire ce rapport unilatéral entre le signifié sonore et le signifié photographique et permettait d'établir un autre rapport au temps et au montage. Une photographie c'est un instant, deux photographies c'est déjà une histoire. Un cliché c'est un monde en soi, deux clichés c'est un monde en suspens.

L'élaboration de ce système de défilement manuel par paires s'est faite au fil de nombreux allers-retours entre la réalisation de photographies et la recherche d'une forme et d'une interface de présentation et de montage. Nous avons réalisé plusieurs portraits « pilotes » qui nous permettaient de tester et de déterminer le nombre de paires ainsi que leur contenu (rapports entre les valeurs de plans, dynamique des flous et des décadrages, jeux sur les jointures etc.).

Par ailleurs, afin de mieux partager l'expérience sonore vécue et décrite par la personne nous avons eu l'idée d'une deuxième bande son qui permette d'écouter les sons évoqués dans l'entretien (les bip-bip de l'hôpital, le brame du cerf, les sonnailles...) Ainsi le son subit un peu le même sort que les paires photographiques puisqu'il se trouve lui aussi fragmenté en deux : la face A pour la voix de la personne qui raconte son histoire, la face B pour les sons dont elle nous parle.

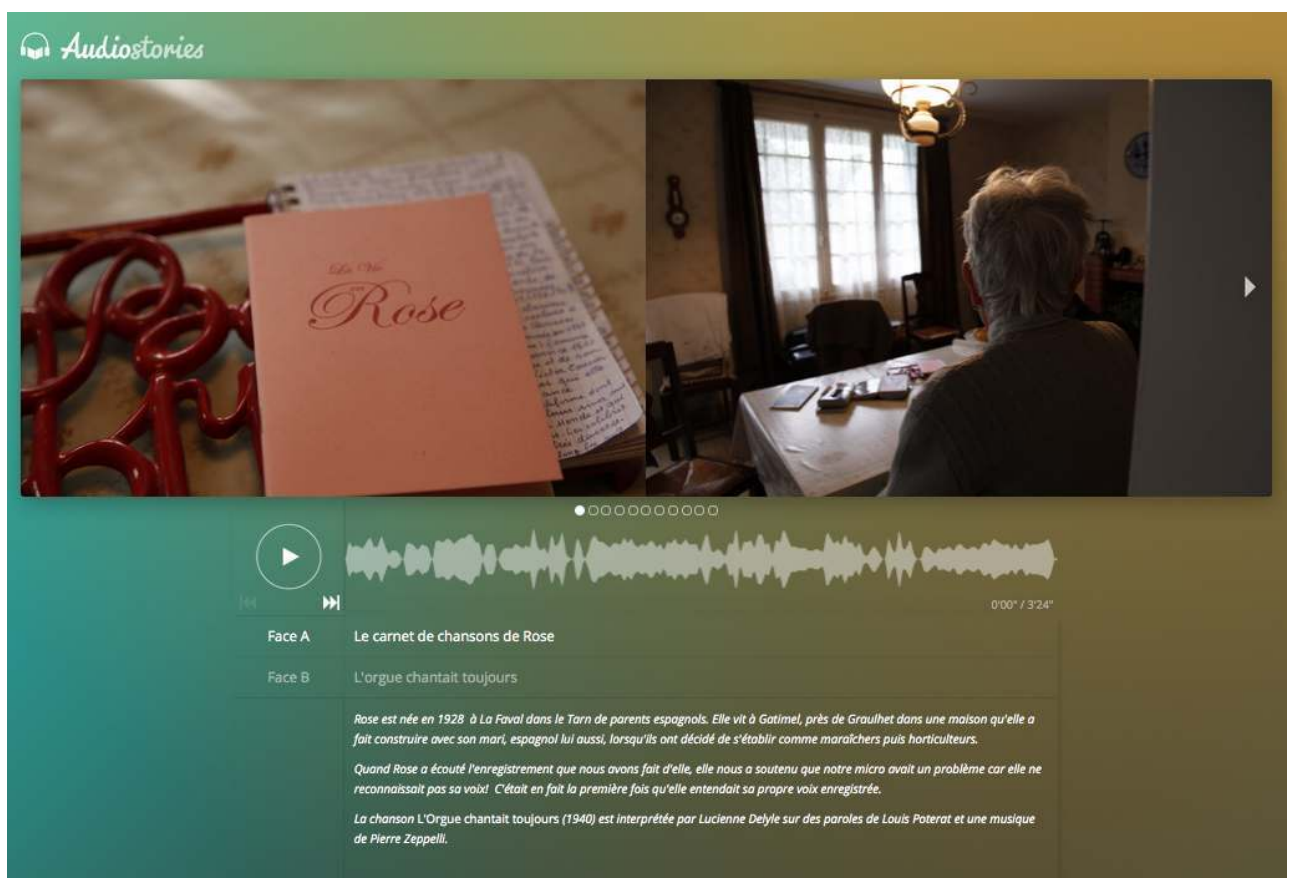

Figure 1 : page d'accueil d'une audiostory 


\section{Une forme diffractée et mobile}

11 L'autre idée principale était d'interrompre le flux du montage image. Ou plutôt de libérer les images d'un défilement qui ne leur appartient pas puisqu'il est décidé par la bande son. Nous avons alors pensé aux visionneuses du début du siècle, ces objets même qui avaient donné l'idée à Chris Marker de réaliser La Jetée. Le film-diaporama préfiguré par Marker en $1962^{3}$ met en avant par sa forme-même la question du temps cinématographique et de la mémoire. Les images fixes viennent ainsi rappeler au cinéma son propre passé, le flux et la fixité renvoyant à l'enjeu crucial d'une présence/absence des fantômes cinématographiques. Dans un texte intitulé " C'était un drôle d'objet ${ }^{4}$ », le cinéaste explique comment le Pathéorama qui permettait de découvrir des films en raccourci et en modèle réduit, lui a non seulement donné envie de créer ses propres images fixes puis ses propres films mais aussi de tordre un peu les frontières entre ces deux régimes d'images. «On ne peut pas faire un film avec des images fixes » lui avait dit un camarade de classe... et trente ans plus tard il réalisait La Jetée !

De cet objet décrit par Marker nous retenons une autre caractéristique: dans le Pathéorama, comme dans d'autres «visionneuses» de la même époque, c'était l'utilisateur qui faisait défiler les « vues » dans le boîtier 5 . Nous avons alors eu l'idée d'un diaporama commandé manuellement qui permette à l'utilisateur de faire ou non défiler les photographies à son propre rythme. Ainsi les rapports entre la bande son et les images sont en quelque sorte laissés au hasard puisqu'ils dépendent entièrement du geste de chaque utilisateur. Il est aussi tout à fait possible de ne pas faire défiler les images pendant l'écoute et de les regarder par la suite. L'expérience est donc toujours unique et toujours « différée ». La non simultanéité entre l'expérience visuelle et sonore est mise en avant. On peut bien sûr choisir d'imbriquer momentanément les images et les sons mais leurs rythmes demeureront toujours autonomes et indépendants : un flux d'un côté et un défilement de l'autre, une continuité sonore d'une part et une intermittence visuelle d'autre part.

13 Ces variations des temporalités avec tout ce que cela implique de rapport à l'immédiateté, à la simultanéité nous rappellent les analyses de Jean-Paul Fargier et plus particulièrement son «effet Tivi» qui permettait de décrypter la tension entre le « direct » de l'expérience télévisée et le «différé » de l'expérience cinématographique ${ }^{6}$. Qu'en est-il de l'expérience du web? Entre archivage et publication journalière, le web n'est-il pas en train de construire un nouveau terrain de jeu pour les combats rapprochés entre le «direct » et le «différé »? Un espace-temps «diffracté » qui multiplie les possibles grâce à un prisme nouveau : l'action de l'utilisateur.

14 Pour le projet audiostories nous avons souhaité que l'internaute construise une durée unique, subjective. L'une des caractéristiques fondamentales du cinéma - la succession des images et de sons selon un montage dont le déroulement est figé - est ici mise en question. Le montage se fait en quelque sorte en ligne. À chaque fois qu'un utilisateur regarde et/ou écoute, il réalise son propre montage qui ne sera inscrit/écrit nulle part si ce n'est dans la mémoire de son expérience de spectateur. Le web permet ce «non montage » et cette indépendance des matériaux qui se voyaient autrefois figés et agglomérés dans les supports tangibles traditionnels (pellicule, cassette...). comparées à des partitions de musique qui seraient jouées par différents « interprètes ", 
ce terme nous paraissant plus adapté à l'expérience esthétique que nous souhaitons proposer que celui d'utilisateur. Par leur forme ouverte les audiostories veulent à leur façon offrir «à l'interprète une œuvre à achever"». Elles s'apparentent peut-être plus encore à ce qu'Eco appelait une "œuvre en mouvement", cette sous-catégorie qu'il distinguait pour les ouvrages mettant en avant leur caractère fragmenté et mobile ${ }^{8}$. Par ailleurs, l'expérience ouverte et mobile des audiostories peut être prolongée de manière encore plus personnelle car après avoir manipulé l'objet, l'interprète peut se l'approprier et écrire grâce à lui ses propres « partitions » audiovisuelles.

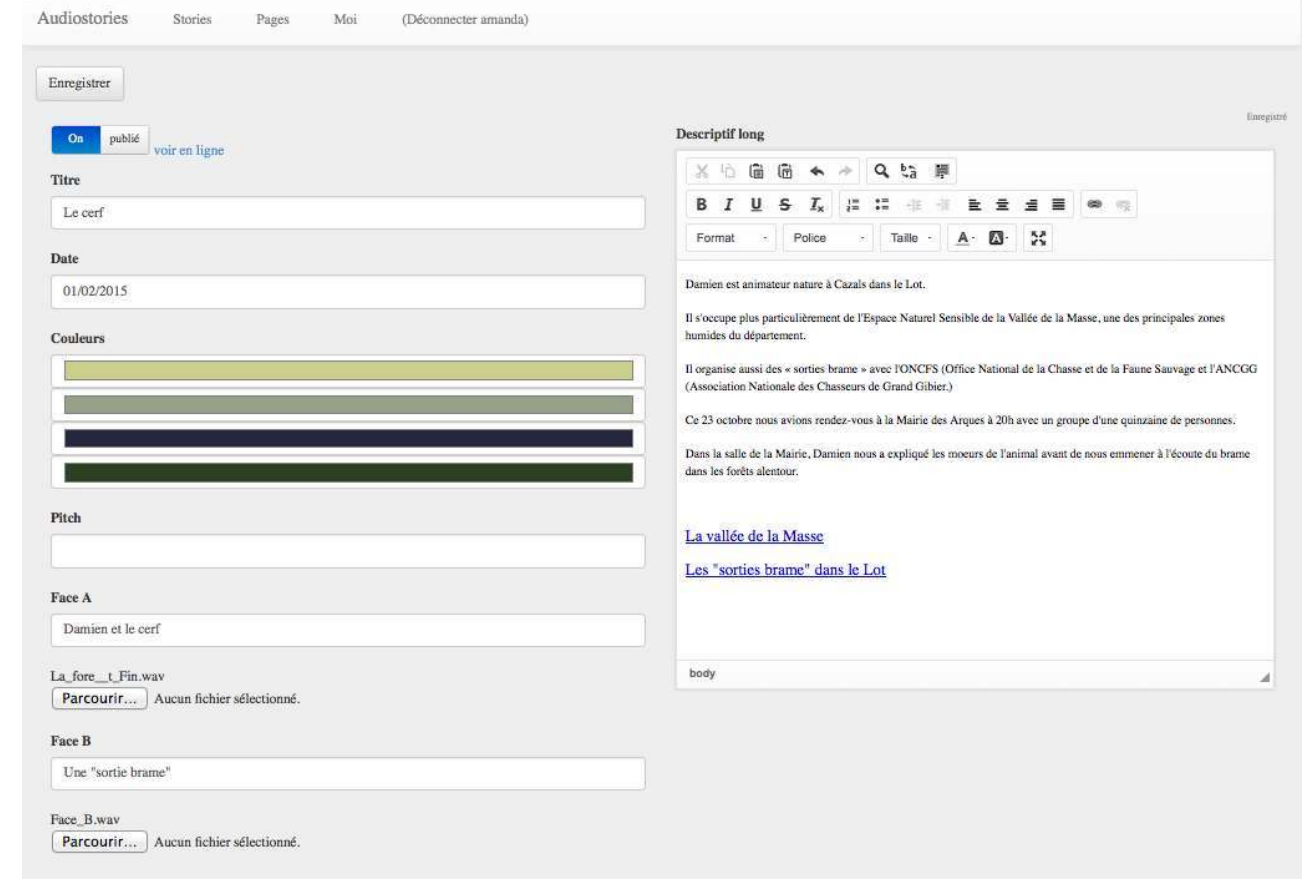

Figure 2 : partie supérieure de l'interface administrateur permettant de gérer les sons, textes et couleurs 


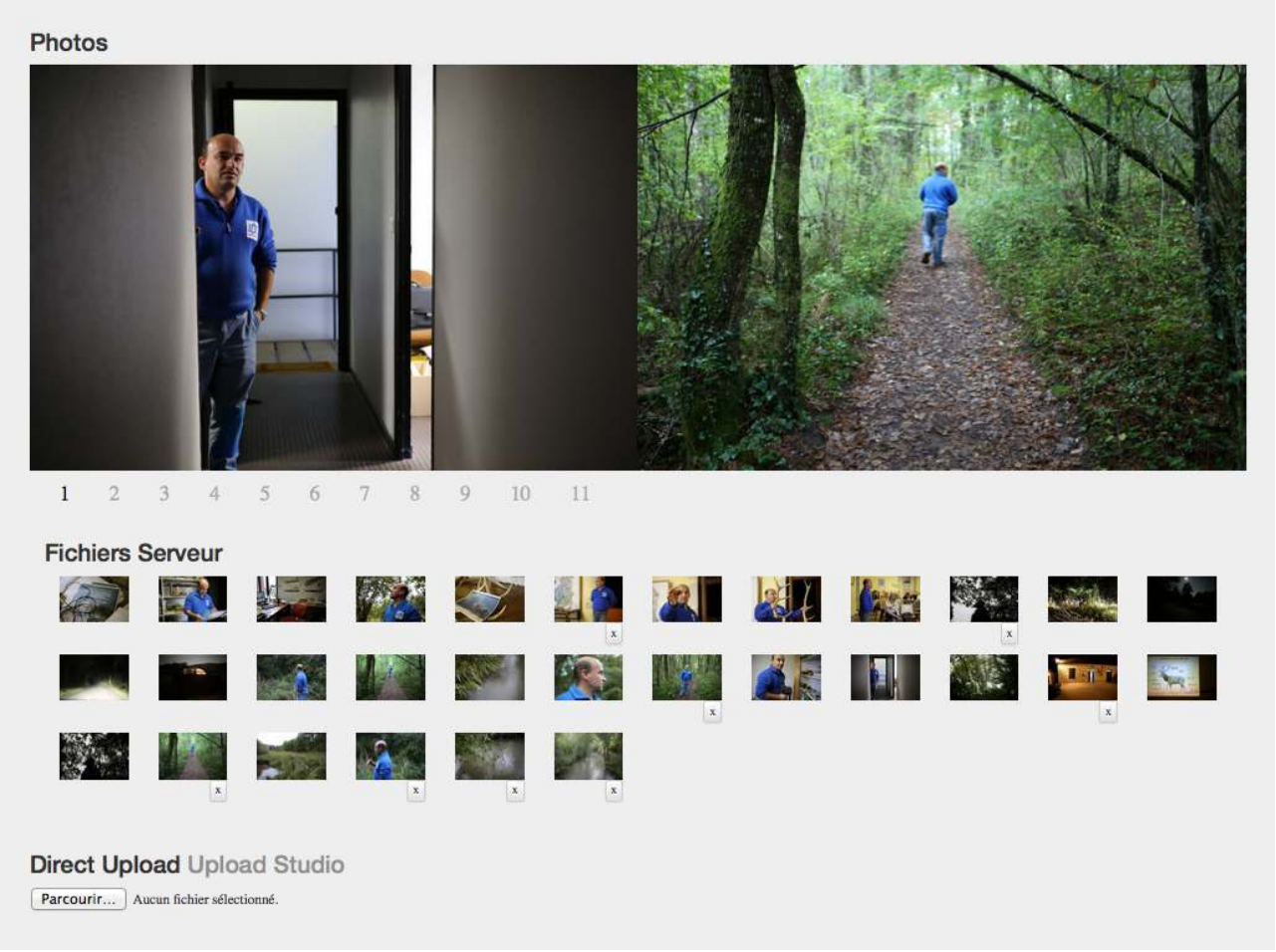

Figure 3 : partie inférieure de l'interface administrateur permettant de gérer les paires photographiques

\section{Partition et partage}

16 L'origine des mots est bien la même et Alain Rey nous indique que le terme partition «a d'abord été employé avec le sens de participation ${ }^{9}$ ». Ainsi, notre volonté de créer des partitions audiovisuelles a pris au fil du temps de nouvelles formes engageant chaque fois davantage la participation de l'interprète. Il s'agissait d'abord, comme nous l'avons expliqué, de laisser libre l'interprétation mais aussi, en présentant un objet audiovisuel "partitionné », de montrer en quelque sorte l'envers du décor, de dévoiler le travail audiovisuel en train de se faire. En choisissant de rendre visible la bande son et en la positionnant au-dessous des photographies nous avions dès l'origine envie de construire un objet audiovisuel inspiré de l'ergonomie des timeline des logiciels de montage vidéo ${ }^{10}$. L'idée était de proposer un objet audiovisuel dont la forme rappelle celle d'un outil. Un objet audiovisuel qui puisse porter en lui la trace même de sa fabrication.

Enfin nous avons souhaité partager aussi cette interface fonctionnant comme une plateforme collaborative de montage en ligne. L'association Le Fil, productrice du projet et qui a aussi travaillé au développement du site et de l'interface travaille depuis plusieurs années à la promotion de la " culture libre », tant dans le domaine des logiciels libres que dans celui du partage de contenus culturels sur internet. Parmi les principes fondateurs du mouvement des logiciels libres, il en est un central que résume parfaitement le précepte d'Eric Steven Raymond : « on ne devrait pas avoir à résoudre deux fois le même problème ${ }^{11}$ ». Ainsi, puisque nous avions créé un nouveau format et un outil de publication en ligne pour ce format, il était évident que nous devions rendre disponible cet outil. Car c'est bien dans la continuité de ce mouvement social ${ }^{12}$ que s'inscrit le projet audiostories, en offrant aux internautes les quatre libertés fondamentales des logiciels 
libres $^{13}$ : la liberté d'utiliser cette nouvelle interface, celle de la redistribuer, éventuellement modifiée et de l'étudier. Distribuer le code source du projet audiostories, c'est pousser la démarche un peu plus loin encore et participer à cette utopie d'un internet où les œuvres circulent librement, s'enrichissent et construisent peu à peu un patrimoine immatériel accessible à tous. Tout comme l'écriture du projet s'appuie sur de nombreuses références cinématographiques et documentaires, sa réalisation technique s'appuie sur tout un corpus de logiciels libres, en premier lieu les systèmes qui font fonctionner internet (Linux, Apache, PHP, etc.) puis des projets plus récents qui animent le web d'aujourd'hui, comme par exemple le projet Angularjs.

Ainsi les réalisateurs souhaitant participer au projet peuvent aujourd'hui construire directement en ligne leur propre audiostory en accédant grâce à un mot de passe à une interface de montage photo/son/texte. Par ailleurs ce CMS (Content Management System) à code source ouvert peut être utilisé pour la création d'autres projets audiovisuels pour le web qui désirent associer sons et photographies. L'interface à partager se veut aussi évolutive avec la possibilité de créer de nouvelles fonctionnalités. Première implémentation d'un CMS Open source, audiostories.fr est donc envisagée comme une première étape dans la recherche de nouveaux outils audiovisuels pour le web.

Combien y aura-t-il d'audiostories dans un an? A quel rythme et par qui seront-elles publiées? Quels « interprètes » viendront jouer ces « morceaux » de documentaires sur le site? Quels bricoleurs s'empareront de notre outil pour proposer d'autres expériences? Nous espérons que ce texte saura donner envie au lecteur de participer à l'expérience et de nous aider à enrichir le site de nouvelles histoires.

Ce projet a soulevé beaucoup plus de questions et nécessité davantage d'expérimentations que nous ne pensions au départ. Maintenant que nous approchons $\mathrm{du}$ but, il nous semble important de dire combien la création audiovisuelle pour le web est un terrain d'expérimentation riche et fascinant parce qu'elle convoque une remise en question à tous les niveaux : durée, media, montage, production, diffusion, financement, auteur/spectateur...

21 Nous avons eu la chance d'être accompagnés au cours de ce projet par deux développeurs web, Fabrice Lapeyrere et Frédéric Jean ainsi qu'un ergonome-designer, Vadim Bernard, qui ont su être à l'écoute de nos tentatives parfois maladroites et nous ont guidés habilement dans nos choix. Si nous signons aujourd'hui ce texte avec Fabrice Lapeyrere c'est pour revendiquer que la création d'un webdoc peut-être tout autant l'oeuvre du développeur que celle des auteurs à l'origine du projet. Ainsi le site et la création de ses contenus sont, eux aussi, nés sous le signe du partage et de la création collective.

Le plus fort potentiel du web réside peut-être avant tout dans sa générosité, sa capacité à accueillir les élans créatifs de chacun. On pourrait alors y voir la possibilité d'une véritable action collective, et non pas simplement la prétendue liberté d'une interactivité qui semble bien souvent fonctionner à vase clos. En effet le plus souvent le terme « interactivité » désigne une action-réaction d'ordre individuel alors que nous aurions envie de lui donner un champ d'action plus large. Défendre l'échange plus que l'interaction, le partition plus que la participation et faire ainsi de l'internaute autre chose qu'un simple utilisateur, mais un interprète des formes mobiles et ouvertes du web. 


\section{NOTES}

1. Comme l'explique Nicolas Bole dans son article "Choses entendues sur le webdoc», toute proposition de « délinéarisation » implique que l'objet premier soit linéaire à l'origine. Le webdoc existe-t-il ? , Le Blog documentaire éditions, janvier 2014, p.40

2. http://interviewproject.davidlynch.com. Dans un article de Libération, David Lynch décrit ce projet comme " un road trip où des gens trouvés au hasard sont interviewés», http://ecrans.liberation.fr/ecrans/2009/06/05/interview-project-un-road-trip-en-ligneproduit-par-david-lynch_959086

3. Selon le Robert historique de la langue française le terme « diaporama » est apparu en 1965 donc trois ans après la sortie de La Jetée.

4. http://chrismarker.org/chris-marker/cetait-un-drole-dobjet-by-chris-marker Ce texte est aussi paru dans le livret du DVD La Jetée - Sans Soleil, ARTE, 2003.

5. Les vues étaient alors présentées par paires quasi identiques pour procurer une sensation de relief.

6. Nous renvoyons ici à l'ensemble de textes réunis dans Ciné et TV vont en vidéo (avis de tempête), De l'Incidence Editeur, Le Havre, 2010, 322 p.

7. ECO Umberto, L'CEuvre ouverte, éditions du Seuil, Paris, 1965, 313 p.

8. Umberto Eco définit les oeuvres en mouvement comme des œuvres matériellement inachevées. En musique, Eco cite le Scambi de Pousseur, en arts plastiques les mobiles de Calder ou les travaux de Bruno Murani.

9. REY Alain, Le Robert, dictionnaire historique de la langue française, Paris, 2012.

10. De la même manière que nous avions conçu un espace original pour présenter les photographies par paires, nous avons élaboré un player son avec une waveform un peu atypique. Ses lignes plus arrondies que celle des player habituels nous semblaient donner une impression plus juste du ressenti face à la rondeur des voix et accentuer la fluidité de la bande sonore.

11. "No problem should ever have to be solved twice», How to become a hacker, Eric Steven Raymond (http://www.catb.org/esr/faqs/hacker-howto.html)

12. http://fr.wikipedia.org/wiki/Culture_libre

13. http://www.gnu.org/philosophy/free-sw.html 